\title{
Framing Real-Life Experimentations as Case Studies
}

\author{
Stefano Crabu, Ilaria Mariani, and Felicitas Schmittinger
}

The chapter describes the case studies methodology on the ground of the volume: their use and comparison are investigated from a theoretical point of view. This chapter has a twofold aim: (i) contextualise case studies and the experimentation/prototyping conducted by the pilots, then (ii) to provide a compass for going through the next chapters in which it is detailed the experience of each pilot as a case study. This reasoning is a premise for understanding and situating the relevant points emerged in the larger picture of the RRI framework.

\section{Introduction}

This chapter has the purpose of presenting the overall methodological framework in which the volume is rooted. It is aim to discuss the case study approach adopted for orienting the production of self- and reflexive narrations about ten RLEs carried out by as many pilot organisations across Europe (Fab Labs, Living Labs and Science Centers and Museums) engaged in addressing relevant societal challenges entangled with various STI domains. In doing so, a meaningful methodological compass is provided for understanding the rationale and the structure of the next ten "empirical chapters". More in detail, the following chapters are consecrated to discuss each "pilot experimentation" as a case study, which allow to critically present, analyse and

\footnotetext{
S. Crabu $(\varangle) \cdot$ I. Mariani · F. Schmittinger

Department of Design, Politecnico di Milano, 20158 Milan, Italy

e-mail: stefano.crabu@polimi.it

I. Mariani

e-mail: ilaria1.mariani@polimi.it

F. Schmittinger

e-mail: felicitas.schmittinger@polimi.it
} 
assess the effectiveness of the adopted co-creation approaches, processes and tools (see Chap. 2). Thus this chapter serves as a methodological premise for clarifying how data from the ten RLEs, in the form of self-narrative case studies, has been gathered allowing: (i) a deeper understanding of the major dimensions at stake in co-creation practices within STI domains and; (ii) a comparative analysis of these major dimensions within the context of the RRI frame.

\section{The Case Study Approach}

As mentioned above, a methodological frame was adopted according to which each RLE has been framed as a case study. The heuristic power of the case study approach is well recognised in different fields, such as social research, design, law and policy, due to its potential for eliciting in-depth, multi-faceted explorations of complex issues in their real-life, naturalistic settings. According to Yin [1], a case study can be defined: "as an empirical inquiry that investigates a contemporary phenomenon within its real-life context; when the boundaries between phenomenon and context are not clearly evident; and in which multiple sources of evidence are used." In this sense, the case study approach is one of the most relevant research strategies to employ for producing an in-depth and thorough appreciation of an event or phenomenon of interest occurring within its natural real-life context. In research, the case study approach can be mobilised, for example, to describe in details patient-physician relationships within different hospitals and how the mutual consent is shaped; or how different practitioners in high-tech firms cooperate for developing an innovative technological solutions for monitoring the air quality; or again to investigate causal links and pathways emerging by the implementation of a new regulatory initiative, or a public service in a concerned geographical area. As a rule, a case study framework selects a small geographical area or a limited number of organisations, or social groups to be scrutinized. Thereby, the case study approach allows a researcher to closely examine data within a specific context. So, case studies enable exploration and investigation of both ongoing real-life processes by means of contingent analysis of specific settings of interactions, and how interactions and conditions under study can influence, and are influenced by the cultural, economic and political landscape.

\section{Eliciting Experiential Knowledge on Co-creation in STI Policymaking}

A case study approach was adopted with the aim to investigate real-life co-design and co-creation practices in STI as a way to (re)shape the missing links between strategic objectives (to make research and innovation more "responsible"), topics and communities (domains of science and technology, groups of stakeholders, citizens 
and society at large), and the activities on the ground (research and innovation). This approach allowed to generate data and information around the "how", "what" and "why" questions at different levels (i.e. national, regional and local), and about different dimensions (i.e. economic, political and social). For example, it opens up reasonings about questions such as "how pre-existing culture of engagement and dialogue between citizens and stakeholders influenced the experimentation". This can support both in developing and refining fresh knowledge about the current forms of public participation in STI policymaking and beyond, as expected within the RRI frame. However, it is worth noticing that a case study is not aimed at exploring an entire organisation. Rather, the analytical gaze focalised on particular issues, by framing the specific RLE as the unit of analysis. This approach allows to understand the complexity of the RLE, by carefully designing and implementing what was called "the self-narration guidelines' (see Sect. 4). This tool enables the production and consistent organisation of the experiential knowledge shaped by the different kinds of practitioners engaged within the concerned RLE, e.g. designers, science communicators, engineers, students, patients and lay people in general. By means of the self-narration guidelines it was aimed at generating "thick description" [2] of what is going on within the experimentation. This work can be considered primarily as an observation activity of ordinary practice occurring in a specific setting. More critically, it is a reflexive activity oriented at producing a thorough account about the multiverse co-creation activities, thus to make sense of local meaning and local knowledge, and relating them to the broader organisational, social, political and economic context. This is provided by the fact that this self-narration casework is based on the direct participation of the authors in the real-time experimentation, spending extended time on site, personally organising co-creation activities of the case, reflecting and revising the descriptions of what is going on. Therefore, it is an analytical and reflexive effort aimed at understanding what is important about the specific experimentation within its own environment, which is peculiar and different for each case. The goal set by the self-narration guidelines is not to describe data as they occur during the RLEs; but to produce a detailed emic account able to provide actionable and analytical insights about how the co-creation experimentation took place, in its different phases, such as the definition of the challenge to be addressed and the process of designing the solutions.

As it will clearly emerge in the next section, in designing the self-narration guidelines specific attention was paid to the mutual engagement between the situated and specific practices for conduction the RLE, and the broad economic, political and social contexts. As a consequence, practitioners were asked to clarify regulatory landscapes and social values and beliefs that entered as a relevant dimension in the course of the experimentation. It is worth noticing that this strategy engendered complex relationships. Indeed, the self-narration guidelines pull attention both to the situated ordinary practices and experience of the practitioners and stakeholders engaged in the RLE and also to the broad large socio-political and regulatory contexts in which each experimentation is located. In this way, self-narration orients to complexities connecting ordinary practices of co-creation occurring in specific settings of interaction to some more broad concerns related to the regulatory and societal environments. 
Thus, in this approach the self-narration guidelines enabled the consideration of the case study both as a process of learning about the specific RLT and the product of the learning produced in SISCODE. Under the aegis of this methodological approach firstly the RLEs are considered as a bounded system that allows to capture specificities at stake in STI co-creation around certain societal challenges developed according to the RRI. Furthermore, the self-narration guidelines work as an "instrumental case study", aimed at highlighting the specific methodological choices, the tools mobilised in the experimentation, and its interpretations in relation to the specific context in which the RTE has been performed.

Finally, in the last chapter the 10 case studies will be analysed as a whole, or as a "collective case study" [3] in order to develop a comparative investigation that can lead to a better understanding of co-creation processes in relation to the STI policymaking. This strategy offers an in-depth and comprehensive understanding of co-creation in Europe, and across different STI domains (such as health, ICT and environmental issues) as a bottom-up and design-led phenomenon together with its corresponding suitable framework conditions. In this way it is aimed to analyse and compare the outcome and condition of the RLEs under scrutiny, thus to assess the result of the impact of co-creation in STI policymaking in relation to the RRI frame. In doing so, the results of the comparative analysis (Chap. 14) will provide insights on suitable strategies for coping with the limit of the current implementation of cocreation in STI policy. Therefore, the comparative analysis is carried out according to the following dimensions:

i. phases of the engagement process they support (i.e. research, Conceptualisation, development, prototyping and testing, assessment);

ii. $\quad$ expected output (i.e. opinions, feedbacks, ideas, product, and service);

iii. sectors of application (i.e. private, public, and third sector);

iv. typology of innovation (i.e. technological, social, scientific, and business).

Overall, innovative knowledge is offered on what works and what does not work to boost the operationalisation of RRI through co-creation.

\section{The Self-narration Guidelines: Rationale and Layout}

The reasoning that follows stems from the awareness that the RRI field reports a general lack of a learning framework aimed at supporting the validation and replication of virtuous mechanisms of co-creation for RRI. In such a context, gaining understanding on how to cope with constraints and barriers that frequently come about along the process constitutes relevant knowledge that can contribute to the successful result of other initiatives.

As stated in the previous paragraph, the basic concept of creating guidelines is based both on the concept of having the participants of the RLE themselves narrating the cases, as well as aligning different pilots in terms of typology of organisation, domains and addressed challenge, thus to make them comparable to some extent. 
Moreover, introducing a unique format shared among the actors engaged in the RLEs paves the way for mutual understanding, contributing in building useful knowledge and consistent narrations about the processes of experimenting.

Exploiting their extensive knowledge of the process, the guidelines are meant to encourage those who compile them-namely the team involved in the co-creation within the labs - to describe their experience as a case considering all fundamental aspects while self-reflecting during the writing.

Given these premises, the objective of asking the team of each RLEs to represent their experimentation through the practice of self-narrations built upon the same guidelines is twofold.

At first, the pilots should have the possibility to narrate their co-creation journey themselves as protagonists of the process, without too much influence of third parties but providing a direction on the desired outcome. This has not only the scope to create a purely first-hand report from the people being directly involved in the experimentation, but also stimulate self-reflection during the writing activity itself. As a matter of fact, the reflective activity is valued that reaches across the process of writing as a moment of fundamental learning per se. On the other hand, providing guidelines as a general layout with key points and questions as an orientation is a way for aligning the very diverse pilots in a similar form, making their process and experiences to some extent comparable to each other. Notwithstanding their diverse background and context, and the fact that each lab focused on different challenges/experimentations, providing them with the same basic structure to follow was key for opening up comparison and critical analysis, nurturing a discussion that goes beyond the singular cases.

Therefore, the guidelines are the result of a methodological process applied to gather information on some aspects fundamental for the experimentation.

In the following the layout is reported as an index, anticipating that each part will be laid out later on sharing the rationale on their ground.

1. Synthesis of the pilot's journey.

2. Initial context.

2.1. External context and ecosystem.

2.2. Organisational background.

3. Challenge.

4. The co-creation process of the envisioned solution.

4.1. Context analysis.

4.2. Problem framing.

4.3. Envisioning solutions.

4.4. Developing and prototyping.

4.5. The role of policies and policymaker engagement.

5. The Final Solution.

5.1. Final concept. 


\subsection{Sustainability strategy.}

6. Transformations triggered and outcomes.

7. Conclusive reflections.

8. References.

In addition to this index as a basic guideline, every section unpacks into key points referring to the desired content and contains a few questions aimed at triggering a detailed and in-depth description of the experimentation, while further stimulating reflection during the writing.

For example, in the final chapter on conclusive reflections, one of the questions had been "Did you come across some unexpected opportunities that you weren't aware of?" to invite the pilots to a broader reflection on alternatives and opportunities identified during the process.

The logic of the layout roughly follows the general co-creation journey that each lab underwent during the experimentation process (see Chap. 2), hence starting from the analysis of the context to the phase of developing and prototyping of the solution. As previously mentioned, the layout is directed towards the collection of specific information related to the main dimensions explored, namely the implementation of RRI in practice, the exploration of capacity building through co-design and prototyping as an approach to transform ideas into implementable solutions. Such dimensions and their enquiry were also carefully inspected during the desk research conducted in the first year of the SISCODE project, and consisting in an extensive literature review and an analysis of existing co-creation cases across Europe (n:138). This preliminary study grasped the potential of co-creation approaches, RRI practices and policies, and their cross-fertilisation to inform the experimentation on the dynamics and outcomes that spurred form of integrating society in science and innovation in a long-term perspective.

As a matter of fact, while RLEs benefited from the investigation of the state of the art regarding practices on co-creation in contexts, as well as from the knowledge base generated in such an enquiry to enrich their processes [4-6], the hereby presented guidelines leaned on such scholarship for defining the dimensions to specifically vet through its self-narrative approach.

Considering the overall objective of delivering insights into the use of collaborative approaches for RRI and policymaking, the analysis of RLEs as case studies needs to keep in mind that a successful implementation of co-creation strongly depends on the interaction with the context [7]. Such interaction has a high degree of complexity, since it is characterised by multilayered social dimensions on various levels. Grasping its logics is primary for a more precise understanding of the dynamics triggered in the ecosystem, as well as their opportunities and barriers [8,9]. These can be attributed to three levels related to as many scales. The macro-level identifies a "process of change in the social structure of a society in its constitutive institutions, cultural patterns, associated social actions and conscious awareness" [10]. The meso-level refers to the intermediate structures as interactions with organisations and alliances. Finally, the micro-level covers the individual scale of the person, its needs and role-conflicts, 
and it allows to understand "how stakeholders and their everyday practices interact with environmental factors" [4].

To gain such an accurate knowledge, the guidelines pose specific attention to the exploration of the context of dependency, the way in which stakeholders are involved, the co-creation practices operated, and the transformations triggered, from the dimension of the team to at an organisational scale.

Table 1 unpacks the question starting from the overall goals of the experimentation, to their sub-elements, up to the link to the dimensions explored.

\section{Context dependency}

Context and its specificities constitute a structural factor to consider when dealing with co-creation and RRI, since it reflects established cultures, mindsets, practices, and policies characterising the specific environment [11]. Since co-creation practices take place in contexts as ecosystems that contain actors with their specificities and inter-dependencies, their understanding can highly impact the success of an initiative. Therefore, introducing this dimension is a way for asking labs to describe and reflect on the context where the experimentation is taking place. Taking this into high consideration means gaining understanding about the networks and partnerships the initiating body upholds, as well as about local culture, structures and policies. As its importance is meant to instruct the self-narrative of the labs, so it also exert its influence in terms of tools. When creating the toolbox (see Chap. 2), the recognised presence of extremely diverse contexts led to the need for modular and customisable tools and activities. The inherent heterogeneity and diversification of contexts had been identified as one of the barriers to the implementation of RRI. In consequence,

Table 1 Overall goals of the experimentation, sub-elements, and dimensions explored

\begin{tabular}{|c|c|c|}
\hline Goals of the experimentation & Details & $\begin{array}{l}\text { Dimension explored in case } \\
\text { studies }\end{array}$ \\
\hline \multirow[t]{3}{*}{ Fill the identified RRI gaps } & $\begin{array}{l}\text { Complexity of societal } \\
\text { problems }\end{array}$ & Context dependency \\
\hline & Engagement of stakeholders & $\begin{array}{l}\text { Context dependency } \\
\text { Stakeholder involvement } \\
\text { Co-creation practices }\end{array}$ \\
\hline & Tangibility of RRI projects & $\begin{array}{l}\text { Context dependency } \\
\text { Stakeholder involvement } \\
\text { Co-creation practices }\end{array}$ \\
\hline \multirow{2}{*}{$\begin{array}{l}\text { Make the single tools modular and } \\
\text { customisable } \& \text { test their } \\
\text { functionality }\end{array}$} & Context matters & Context dependency \\
\hline & Tools appropriation & $\begin{array}{l}\text { Context dependency } \\
\text { Co-creation practices } \\
\text { Capacity building and } \\
\text { organisational change }\end{array}$ \\
\hline \multirow{2}{*}{$\begin{array}{l}\text { Trigger reflexivity through the use } \\
\text { of tools }\end{array}$} & Comparison necessities & Context dependency \\
\hline & Common knowledge spaces & $\begin{array}{l}\text { Co-creation practices } \\
\text { Capacity building and } \\
\text { organisational change }\end{array}$ \\
\hline
\end{tabular}


several tools were inserted in the toolbox aiming at encouraging to explore the influence of this dimension, valuing the surrounding context specifically relevant and its investigation in the policy context. In parallel, specific attention is drawn on how tools and methodologies are adopted individually by each lab in relation to the environment, as well as differences and similarities in regard to barriers and opportunities identified in diverse contexts.

\section{Stakeholder involvement}

The engagement and constant relationship with concerned actors is crucial both in codesign and RRI. Considering the relationship between the context where the problem is situated and the network that will co-create the solution is central [12-14]. Especially in co-creation processes, the interaction between people with different cultures, backgrounds and forms of knowledge within a frame of collaboration enables the opportunity for both conflict and a learning process where knowledge is shared among peers. Knowledge and expertise lies among different stakeholders, and their involvement enables them to grasp complementary and critical insights. Therefore, it becomes fundamental to identify the various stakeholders groups and local actors to be actively involved throughout the entire process. Being it simple user experience, social knowledge or 'expert' technical knowledge, the benefits from engaging the public goes beyond the verification of hypothesis. Relevant advice, then, regards the possibility to extract both behavioral schemes and best practices from their various domains of knowledge. Public participation is a way to recognise and value their motivation, needs and behaviors, as well as a way to develop context-based solutions [7].

Moreover, recognising that policymakers often do not value social knowledge as equal or valuable as 'expert' technical knowledge [7], the experimentation specifically focused on the inclusion of this group of stakeholders. Investigating possible interplays and interactions by involving policymakers along the entire co-creation becomes a way to better frame the context of STI policymaking in particular as one of the core objectives of the study.

Specific aspects to be explored in the analysis are the level of engagement (active or passive), the constancy throughout the various phases and their overall role.

\section{Co-creation practices}

Co-creation as the way to operationalise RRI in this experimentation is inspected under various aspects. On one hand, its general efficiency and efficacy in RRI contexts is to be explored together with the potential need to be adapted and modified to entirely satisfy the needs for its application in an RRI context.

Aspects to consider in this dimension are its changeableness and potential to be modified for specific contexts and situations, and how this variability can be communicated minimizing the risk of being too broad and open hindering the actual adoption. Finding this balance is specifically important for an effective introduction of co-creation. Here it is particularly relevant to reason about the risks that come across skepticism and resistance, especially in fields with very different current practices like policymaking. Ways to deal with this resistance are to be investigated as well. 
Addressing how such aspects have been tackled by going through a process of selfnarration is a way to encourage labs to gain further awareness about their learning, turning them into shareable knowledge.

Particular attention is drawn to the phase of prototyping as the transition from sheer ideas to potential implementable solutions [15]. This is a particularly crucial point to be investigated to evaluate the potential of the design approach to bridge the gap identified in RRI of moving towards real implementation [16].

\section{Capacity building and organisational change}

Co-creation can bring knowledge and assumptions about who contributes in creating solutions and defining policies, also challenging existing or established practices [7]. To ensure a long-term change and a full embedding of the design approach, the capacities related to it need to be fully incorporated into the organisation and its members to be applied successfully and trigger substantial change in the organisation [17].

The specific focus here lies on two kinds of knowledge acquisition. On one hand the capacities built within the organisation and their influence on its culture and practices beyond the project. On the other hand, the capacities acquired by participants that are not members of the organisation like stakeholders or users are investigated. This is relevant to explore the possibilities and methodologies of triggering change in external entities and actors through concrete involvement in a project. In fact, since they introduce practices and tools able to challenge an established order, cocreation and co-design are political acts. In consequence, it is paramount to invite labs to ruminate about the transformations they activated during their co-creation processes, especially focusing on aspects and situations that encountered resistance to change reflecting on potential futures and an outlook on long-term change.

Moreover, this dimension is also meant to encourage reflection about capacities developed along the way, as well as about barriers to capacity building encountered.

\section{Implementing the Guidelines: 10 Experiences of Co-creation}

Examples of realities where new visions and processes of co-design aimed at actively involving stakeholders in the co-creation of solutions and favourable policies and frameworks are flourishing across Europe in innovation labs exploring citizen science like policy labs, Living Labs, Fab Labs or Science Centers and Museums. Within this context, the experimentation has been implemented in three main domains, that of Fab Labs (n:3), Living Labs (n:3), and Science Centres and Museums (n:4).

Recognising that the range of practices depends on the several variables of the complex landscape where co-creation and design take place, innovation labs come to the fore for being spaces where design-led practices are translated into implementable solutions. In particular, they emerge as characterised by a variety of approaches and tools not only adopted but often further developed to meet their needs and 
better answer to local conditions and challenges, showing an inherent openness to experimentation while being adaptive and flexible.

In the following chapters it will be explored how the structured process of selfnarration intended for connecting the practice with the capacity to set up an analytical, reflective and learning framework, encouraged to frame and make the experiential knowledge gathered intelligible. Although they all aim at a better inclusion and participation of society in science, technology and innovation, each experiment presents its own challenge, context, features and peculiarities, as demonstrated and discussed in the following chapters.

\section{References}

1. Yin RK (1984) Case study research: design and methods. Sage, Beverly Hills, CA

2. Geertz C (1973) The interpretation of cultures: selected essays. Basic Books, New York, NY

3. Stake RE (1994) Qualitative case studies. In: Denzin NK, Lincoln L (eds) Handbook of qualitative research. Sage, London, pp. 443-466

4. Eckhardt J, Kaletka C, Klimek T (2019) SISCODE knowledge base. SISCODE deliverable D2.1, https://ec.europa.eu/research/participants/documents/downloadPublic?docume ntIds $=080166 \mathrm{e} 5 \mathrm{c} 1 \mathrm{fca} 367$ \&appId=PPGMS, last accessed 2021/01/21

5. Kaletka C, Eckhardt J, Krüger D (2018) Theoretical framework and tools for understanding cocreation in contexts. SISCODE deliverable D1.3. https://ec.europa.eu/research/participants/doc uments/downloadPublic?documentIds=080166e 5 bed $185 \mathrm{fb} \&$ appId=PPGMS. Last accessed 2021/01/21

6. Smallman M, Patel T (2018) RRI research landscape. SISCODE deliverable D1.1. https:// ec.europa.eu/research/participants/documents/downloadPublic?documentIds $=080166 \mathrm{e} 5 \mathrm{bed} 1$ 7e30\&appId=PPGMS. Last accessed 2021/03/02

7. Rizzo F, Deserti A, Crabu S, Smallman M, Hjort J, Hansen SJ, Menichinelli M (2018) Co-creation in RRI practices and STI policies SISCODE deliverable D1.2. https://ec.eur opa.eu/research/participants/documents/downloadPublic?documentIds=080166e 5bedc3a0d \& appId=PPGMS. Last accessed 2020/11/21

8. Domanski D, Howaldt J, Kaletka C (2020) A comprehensive concept of social innovation and its implications for the local context-on the growing importance of social innovation ecosystems and infrastructures. Eur Plan Stud 28:454-474

9. Kaletka C, Markmann M, Pelka B (2017) Peeling the onion. An exploration of the layers of social innovation ecosystems. Modelling a context sensitive perspective on driving and hindering factors for social innovation. Eur Public Soc Innov Rev 1(2)

10. Zapf W (2003) Sozialer Wandel. In: Schäfers B (ed) Grundbegriffe der Soziologie. Leske + Budrich, Opladen, pp 427-433

11. Howaldt J, Schwarz M (2010) Social innovation: concepts, research fields and international trends. Sozialforschungsstelle, Dortmund

12. Deserti A, Rizzo F (2014) Design and organisational change in the public sector. Des Manag J 9:85-97

13. Deserti A, Rizzo F (2020) Context dependency of social innovation: in search of new sustainability models. Eur Plan Stud 28:864-880

14. Manzini E, Rizzo F (2011) Small projects/large changes: participatory design as an open participated process. CoDesign 7:199-215

15. Blomkvist J, Holmlid S (2011) Existing prototyping perspectives: considerations for service design. Nordes 4

16. von Schomberg R (2013) A vision of responsible research and innovation. In: Owen R, Bessant J, Heintz M (eds) Responsible innovation. Wiley, Chichester, pp 51-74 
17. Junginger S, Sangiorgi D (2009) Service design and organisational change. Bridging the gap between rigour and relevance. In: International association of societies of design research. KOR, pp 4339-4348

Open Access This chapter is licensed under the terms of the Creative Commons Attribution 4.0 International License (http://creativecommons.org/licenses/by/4.0/), which permits use, sharing, adaptation, distribution and reproduction in any medium or format, as long as you give appropriate credit to the original author(s) and the source, provide a link to the Creative Commons license and indicate if changes were made.

The images or other third party material in this chapter are included in the chapter's Creative Commons license, unless indicated otherwise in a credit line to the material. If material is not included in the chapter's Creative Commons license and your intended use is not permitted by statutory regulation or exceeds the permitted use, you will need to obtain permission directly from the copyright holder.

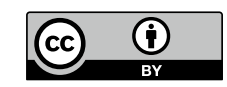

